respectively, and Y. Sakamoto for help with microsequence analysis. This work was supported in part by grants for Scientific Research on Priority Areas, for COE Research, and for JSPS fellows from the Ministry of Education, Science, Sports and Culture of Japan.

Correspondence and requests for materials should be addressed to Y.T.

(e-mail: tsujimot@gene.med. osaka-u.ac.jp). The nucleotide sequence data will appear in the Genbank nucleotide sequence database under the accession numbers AF124726, AF124727, AF124728, AF124725, AF124729 for human acinusL, S, S' and mouse acinusS and $\mathrm{S}^{\prime}$, respectively.

\section{Suppression of Raf-1 kinase activity and MAP kinase signalling by RKIP}

Kam Yeung ${ }^{\star} \dagger$, Thomas Seitz $\dagger \neq$, Shengfeng Li $\$$, Petra Janosch\|, Brian McFerran\|, Christian Kaiser $\ddagger$, Frances Feell, Kostas D. Katsanakis\|, David W. Rose 9 , Harald Mischak\#, John M. Sedivy ${ }^{\star} \uparrow$ \& Walter Kolch $\dagger \|$

${ }^{*}$ Brown University, Department of Molecular Biology, Cell Biology and Biochemistry, Richmond 02912, USA

$\ddagger$ GSF-Research Centre for Health and Environment, Institute for Clinical Molecular Biology, Marchioninstrasse 25, 81377 München, Germany

$\|$ Beatson Institute for Cancer Research, CRC Beatson Laboratories, Switchback Road, Bearsdon, Glasgow G61 1BD, UK

I University of California San Diego, Department of Medicine and Whittier Diabetes Program, 9500 Gilman Drive, La Jolla, California, 92093-0673, USA

\# Franz-Volhard Klinikum, Max Delbrück Centre, Wiltbergstrasse 50,

13122 Berlin, Germany

$\dagger$ These authors contributed equally to this work

Raf-1 phosphorylates and activates MEK-1, a kinase that activates the extracellular signal regulated kinases (ERK). This kinase cascade controls the proliferation and differentiation of different cell types $^{1,2}$. Here we describe a Raf-1-interacting protein, isolated using a yeast two-hybrid screen. This protein inhibits the phosphorylation and activation of MEK by Raf- 1 and is designated RKIP (Raf kinase inhibitor protein). In vitro, RKIP binds to Raf-1, MEK and ERK, but not to Ras. RKIP co-immunoprecipitates with Raf- 1 and MEK from cell lysates and colocalizes with Raf- 1 when examined by confocal microscopy. RKIP is not a substrate for Raf1 or MEK, but competitively disrupts the interaction between these kinases. RKIP overexpression interferes with the activation of MEK and ERK, induction of AP-1-dependent reporter genes and transformation elicited by an oncogenically activated Raf-1 kinase. Downregulation of endogenous RKIP by expression of antisense RNA or antibody microinjection induces the activation of MEK-, ERK- and AP-1-dependent transcription. RKIP represents a new class of protein-kinase-inhibitor protein that regulates the activity of the Raf/MEK/ERK module.

In metazoans the Ras/Raf-1/MEK/ERK module is a ubiquitously expressed signalling pathway that conveys mitogenic and differentiation signals from the cell membrane to the nucleus ${ }^{1}$. This kinase cascade appears to be spatially organized in a signalling complex that is nucleated by Ras proteins ${ }^{3}$. The regulation of the Ras/Raf-1/ MEK/ERK module is complex and may include associations with scaffolding and regulatory proteins ${ }^{4}$. To isolate such proteins we used the Raf- 1 kinase domain, $\mathrm{BXB}^{5}$, as bait in a yeast two-hybrid screen $^{6}$. Screening 500,000 clones of a human T-cell library yielded nine clones that specifically interacted with BXB. Five clones corresponded to 14-3-3 proteins. One clone, RKIP, bound to both kinase-active and kinase-negative $\mathrm{BXB}$, but not to control baits (Fig. 1a). Partial sequencing of the RKIP complementary DNA predicted a protein identical to the phosphatidylethanolamine

§ Present address: Cor Therapeutics Inc., South San Francisco, California 94080, USA a

$\begin{array}{lcc}\text { Bait } & \begin{array}{l}\text { RKIP (His- } \\ \text { prototrophy) }\end{array} & \begin{array}{l}\text { RKIP }(\beta-g a \\ \text { activity })\end{array} \\ \text { BXB } & \text { yes } & \text { yes } \\ \text { Kinase-dead BXB } & \text { yes } & \text { yes } \\ \text { cdk2 kinase } & \text { no } & \text { no } \\ \text { p53 } & \text { no } & \text { no } \\ \text { Lamin } & \text { no } & \text { no }\end{array}$

b
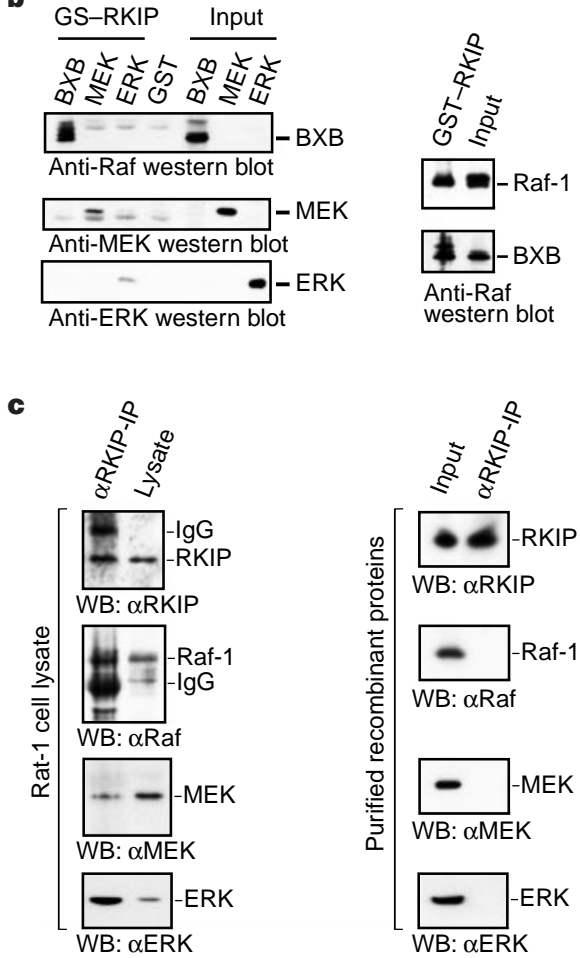

d
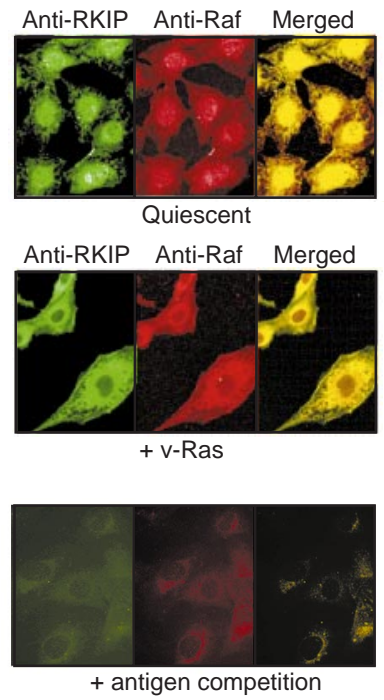

Figure 1 In vitro interaction of RKIP with components of the ERK pathway. a, RKIP interacts with BXB but not with control baits in the yeast two-hybrid system. b. Binding of recombinant BXB, full-length Raf-1, MEK-1 and ERK-2 to GST-RKIP beads. Input: $1 \%$ of the respective proteins used in binding reactions; GST: GST-beads.

c. Co-immunoprecipitation of Raf-1, MEK and ERK with RKIP in Rat-1 cells. The RKIP antiserum does not precipitate recombinant Raf-1, MEK-1 and ERK-2 proteins individually. d, Colocalization of Raf- 1 and RKIP in 208F fibroblasts by confocal microscopy. Antigen competition: antisera were pre-absorbed with their cognate antigens. 
binding proteins (PEBP) with relative molecular mass 23,000 $\left(M_{\mathrm{r}}=23 \mathrm{~K}\right)$ from humans and monkeys. These proteins are widely expressed and evolutionarily conserved, but their functions remain obscure ${ }^{7}$.

Purified recombinant RKIP was tested for binding to the components of the Ras/Raf-1/MEK/ERK cascade. RKIP associated with BXB, full-length Raf-1, MEK-1 and (more weakly) with ERK-2, but not with Ras. RKIP binding was independent of Raf-1 kinase activity, not affected by phosphatidylethanolamine, and direct, as shown by the interaction of purified proteins produced in Escherichia coli (Fig. 1b and data not shown).

These interactions were also shown between endogenous mammalian proteins. An RKIP antiserum co-immunoprecipitated Raf1, MEK and ERK from Rat-1 cells. This was not due to crossreactivity, because the RKIP antiserum failed to immunoprecipitate purified Raf-1, MEK-1 or ERK-2 individually (Fig. 1c). These interactions were also observed in reciprocal immunoprecipitations with antisera to Raf-1, MEK or ERK (data not shown). Confocal microscopy revealed extensive colocalization between Raf-1 and RKIP, in both quiescent and Ras-transformed cells (Fig. 1d).

To investigate the relevance of the interaction between RKIP and the kinases of the Raf/MEK/ERK module in mammalian cells, we inhibited endogenous RKIP by antibody microinjection or expression of antisense RNA. As the AP-1 transcription factor is a major target of Raf signalling ${ }^{8-10}$, we tested the influence of RKIP on AP-1 activity (Fig. 2a). Microinjection of affinity-purified anti-RKIP antibodies robustly activated a co-injected AP-1-dependent reporter gene in serum-deprived Rat-1 fibroblasts (Fig. 2a). This effect was highly specific, because the injection of control immunoglobulin (IgG) was ineffective, anti-RKIP IgG did not affect the expression of a cAMP-dependent reporter gene, and co-injection of an RKIP expression vector abolished AP-1 induction by anti-RKIP
IgG. We also downregulated RKIP expression using an RKIP antisense vector, pAS-C143. This vector markedly reduced RKIP levels without affecting the expression of MEK-1 or actin (Fig. 2b). pASC143 substantially induced the AP-1 reporter gene in serum-starved NIH 3T3 cells (Fig. 2c). These data confirm the microinjection results and show that RKIP suppresses the Raf/MEK/ERK pathway.

Overexpression experiments further confirmed this conclusion. RKIP transfection diminished basal as well as BXB-induced AP-1 activity (Fig. 3a), and microinjection of an RKIP expression vector impaired AP-1 induction by BXB (Fig. 3b). Notably, RKIP did not interfere with AP-1 stimulation by ERK-1. Next, we tested the effects of RKIP overexpression in transformation assays. In contrast to transient reporter-gene assays, transformation assays accommodate the complexity of cellular responses to the chronic deregulation of a single signalling component. RKIP significantly reduced the transformation efficiency of BXB in three distinct assays: morphological transformation, focus formation and anchorage-independent growth (Fig. 3c). RKIP also decreased the total colony yield, albeit to a lesser extent than transformation, showing that RKIP interferes with Raf-mediated proliferation as well as transformation. In contrast, RKIP impaired the induction of foci by $v$-fos or mutationally activated MEK alleles only to a small extent, and failed to inhibit $v$-src transformation (Fig. 3d), indicating that RKIP may specifically block transformation by the Raf/MEK/ERK pathway, primarily by inhibiting Raf.

To investigate the effects of RKIP on individual activation steps, we reconstructed the Raf/MEK/ERK cascade in vitro using recombinant proteins (Fig. 4a). RKIP decreased the phosphorylation of MEK by Raf-1, but did not inhibit ERK phosphorylation by MEK or ELK phosphorylation by ERK. In addition, RKIP (1) failed to inhibit MEK-DD, a constitutively active mutant of $\mathrm{MEK}^{11}$, or MEK activated by TPA treatment of cells (Fig. 4b); (2) did not

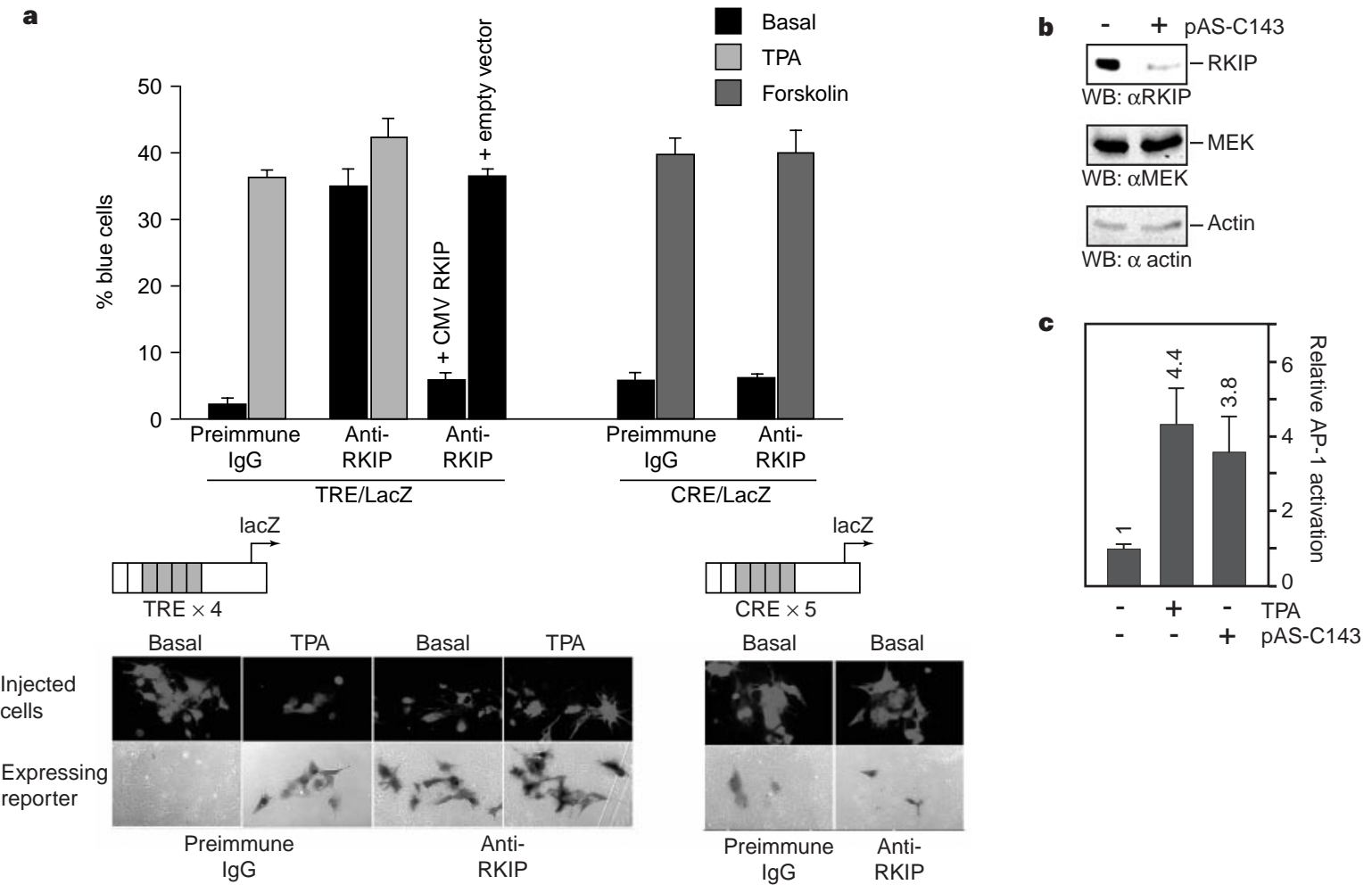

Figure 2 Inhibition of endogenous RKIP activates AP-1-dependent transcription. a, Microinjection of anti-RKIP antibodies. Quiescent Rat-1 cells were microinjected with the indicated reporter plasmids and antibodies and either left unstimulated or treated with $200 \mathrm{ng} \mathrm{ml}^{-1}$ TPA or $20 \mu \mathrm{g} \mathrm{ml}^{-1}$ forskolin. b. The RKIP antisense vector, pAS-C143, downregulates expression of endogenous RKIP. NIH 3 T3 cells were cotransfected with
pAS-C143 and a GFP-expressing plasmid. GFP-positive cells were isolated by FACS and immunoblotted with the indicated antibodies. c, The activity of an AP-1 reporter gene was measured in serum-starved or TPA-stimulated NIH 3 T3 cells following cotransfection with RKIP antisense (pAS-C143) or empty vectors. 
prevent MEK phosphorylation by MEKK-1 (Fig. 4c); and (3) did not interfere with Raf-1 autophosphorylation or phosphorylation of myelin basic protein (MBP) by Raf-1 (Fig. 4d). These data indicate that RKIP is a very selective inhibitor that specifically blocks activation of MEK by Raf.

In vitro, RKIP disrupted the physical interaction between Raf-1 and MEK, which is required for MEK phosphorylation ${ }^{12}$, and behaved like a competitive inhibitor for MEK (data not shown). Therefore, we investigated whether this mechanism also operated in cells. The downregulation of endogenous RKIP expression by the pAS-C143 antisense vector substantially enhanced phosphorylation of MEK on activation-specific sites (Fig. 5a). Similarily, microinjec-
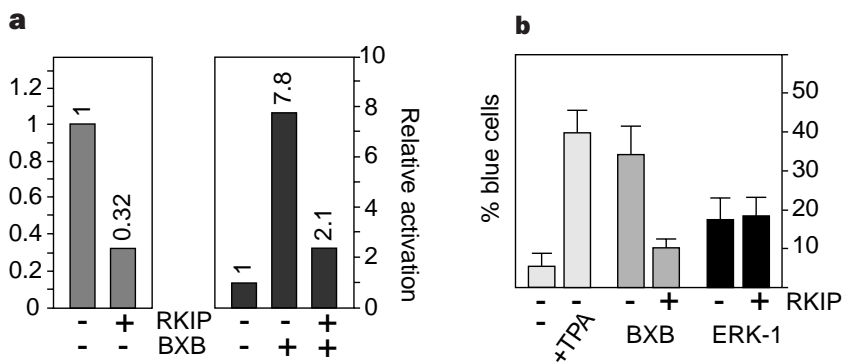

c
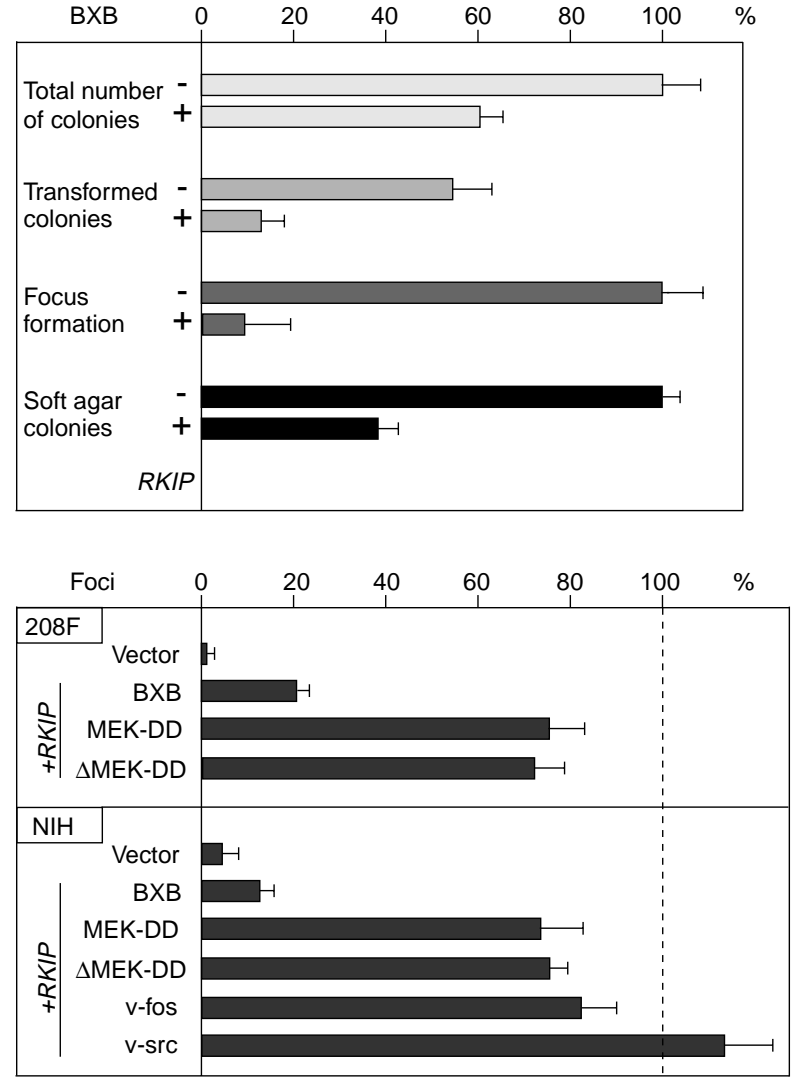

Figure 3 RKIP inhibits Raf-induced AP-1 activation and transformation. a, RKIP reduces basal and BXB-induced AP-1 activity in NIH 3 T3 cells cotransfected with a 3xTRE-CAT reporter and the indicated expression plasmids. b, RKIP blocks BXB- but not ERK-induced AP-1 activation. Rat-1 cells were co-microinjected with a 4XTRE-lacZ reporter and the indicated expression vectors. c, RKIP inhibits Raf-dependent proliferation and transformation. NIH 3T3 cells were transfected with BXB, alone or together with RKIP (linked to neo). G418-resistant colonies were counted and scored for morphological transformation. Aliquots of the same transfection were allowed to grow to confluency without drug and were scored for focus formation. A BXB-transformed cell line was infected with LXSH-RKIP retrovirus or LXSH (hygromycin resistant) and seeded in soft agar in the presence of hygromycin. d, RKIP does not inhibit transformation by $v$-fos, $v$-src or mutationally activated MEK ${ }^{11}$ in $208 \mathrm{~F}$ or $\mathrm{NIH}$ cells. Data are expressed as reduction in focus formation relative to cotransfection with empty vector (set to 100\%). tion of RKIP antibodies enhanced ERK activation in NIH 3T3 cells (Fig. 5b). In a complementary approach, RKIP was over-expressed. Cotransfection of RKIP had only a small influence on the activation of Raf- 1 by TPA, but strongly inhibited the activation of MEK in a dose-dependent fashion (Fig. 5c). EGF produced the same results (data not shown). RKIP overexpression also downregulated the activation of ERKs by the $v$-Ras or $v$-Src oncogenes (Fig. $5 \mathrm{~d}$ ). Cotransfection of increasing amounts of an RKIP expression plasmid inhibited the BXB-induced activation of ERK in a dosedependent manner. In contrast, RKIP did not affect activation by ERK by MEK-DD (Fig. 5e). These data confirm the in vitro results (Fig. 4), and show that RKIP regulates the ERK pathway primarily at the Raf/MEK interface in vivo.

To test whether the association between Raf and RKIP changes during mitogenic stimulation, we monitored the presence of RKIP in Raf-1 immunoprecipitates prepared from Rat-1 cells at different times after stimulation with serum (Fig. 6). The activation kinetics of the ERK pathway closely correlated with a decrease in RKIP coprecipitation. Furthermore, as the activity of the ERK pathway returned to basal levels at later times following mitogenic stimulation, the interaction between Raf- 1 and RKIP returned to the level seen in quiescent cells.

What could be the physiological role of an inhibitor such as RKIP? Like the ERK pathway, RKIP is widely expressed. A quantification of Raf-1, MEK, ERK and RKIP protein levels in the cell lines used in this study showed a wide variation in RKIP expression relative to the kinases. The ratio Raf-1:MEK:ERK:RKIP was 1:1.6:2.4:14 in Rat-1; 1:1.4:3.5:27 in 208F; 1:0.7:9:4.2 in NIH 3T3; and 1:2.9:5.9: $<1.9$ in COS-1 cells. Thus, at least in the three fibroblast cells lines, RKIP is abundant enough to be stoichiometrically relevant as an inhibitor. RKIP also colocalized with Raf-1 in Ras-transformed cells (Fig. 1d), indicating that an appreciable fraction of Raf-1 and its inhibitor RKIP may remain associated even under conditions that promote Raf- 1 activation. This may in part explain the observation that only a small fraction of Raf- 1 can be activated ${ }^{13}$. We propose that RKIP could function like a rheostat that sets the sensitivity threshold for the activation of the Raf/MEK/ ERK pathway. A quantitative analysis of the activation kinetics of the ERK pathway showed that this cascade operates like a switch a

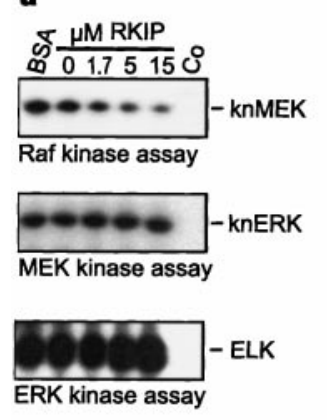

b

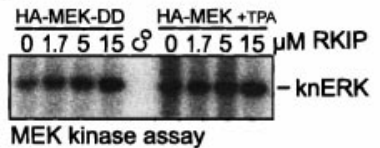

MEK kinase assay

c

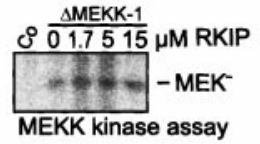

d

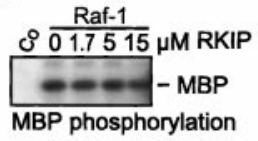

Figure 4 RKIP specifically blocks MEK phosphorylation by Raf-1. a, Effect of RKIP on the activation steps of the Raf/MEK/ERK cascade reconstituted in vitro with purified recombinant proteins. BSA, $15 \mu \mathrm{M}$ bovine serum albumin; Co, substrate alone; $\mathrm{kn}$, kinase-negative mutant. b, RKIP does not inhibit activated MEK. HA-MEK-DD or HAMEK-1 (ref. 11) expressed in COS-1 cells was immunoprecipitated with anti-HA antibodies from serum-starved cells or TPA-treated cells, respectively, and assayed for kinase activity. c, RKIP does not inhibit MEK phosphorylation by MEKK-1. $\triangle$ MEKK-1 (ref. 26) was immunoprecipitated from transiently transfected COS-1 cells and used to phosphorylate knMEK. d, RKIP does not inhibit Raf-1 autophosphorylation or phosphorylation of MBP. 
a

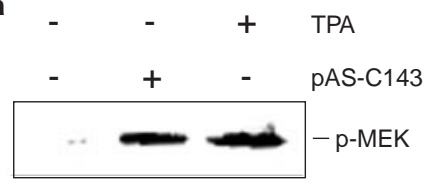

Anti-phospho-MEK blot

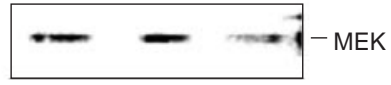

Anti-MEK blot b

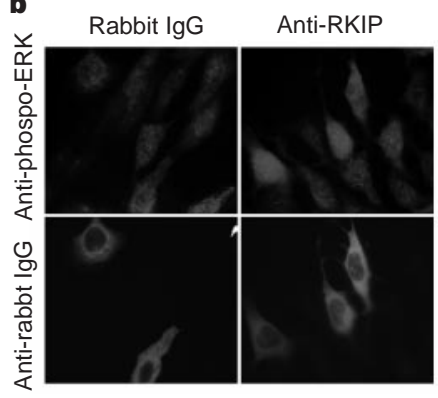

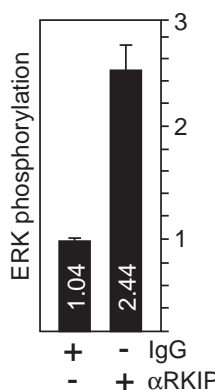

c

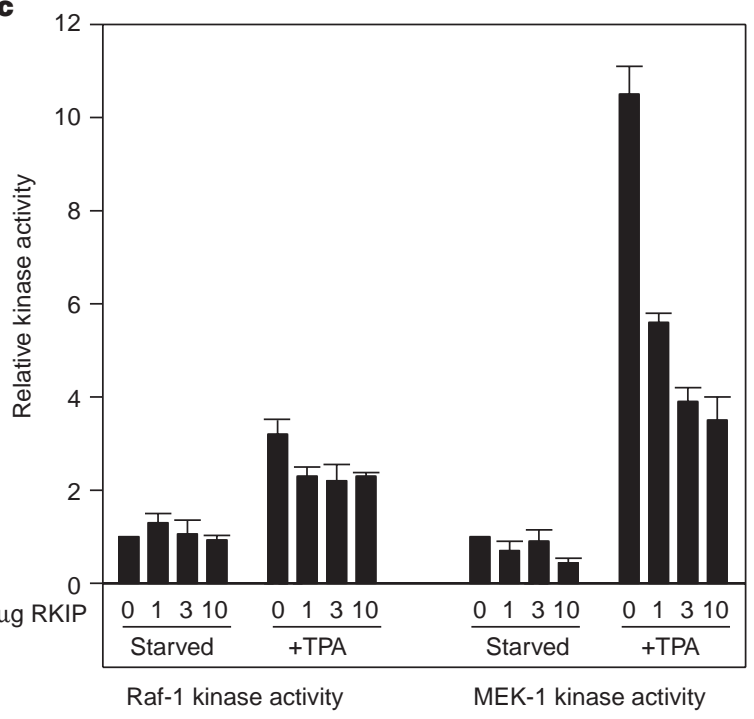

d

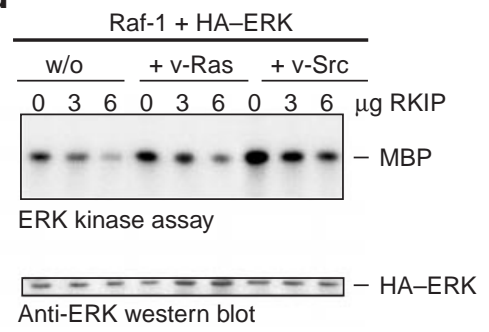

e

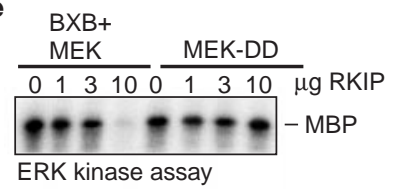

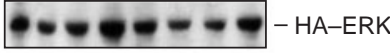

Figure 5 RKIP regulates MEK and ERK activation in vivo. a, RKIP downregulation activates MEK. NIH 3T3 cells were cotransfected with GFP and the RKIP antisense plasmid, pASC143. GFP-positive cells were FACS sorted and immunoblotted with the indicated antisera. b, RKIP antibody microinjection enhances ERK activation. Quiescent NIH 3T3 cells were microinjected with anti-RKIP or control lgG and stimulated with $10 \mathrm{ng} \mathrm{ml}^{-1} \mathrm{TPA}$ for $30 \mathrm{~min}$. ERK activation was visualized with a monoclonal anti-phospho-ERK antibody (Sigma) and quantified densitometrically. c, RKIP inhibits MEK-1 activation. COS-1 cells were transiently transfected with HA-MEK and increasing amounts of RKIP expression

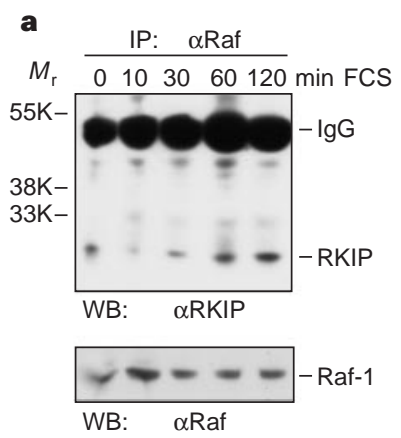

b
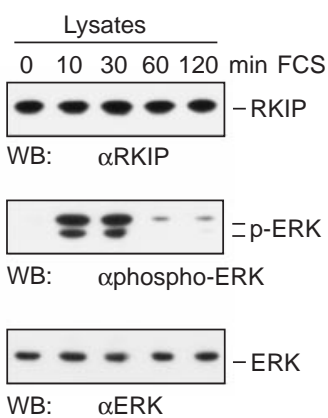

Figure 6 During mitogenic stimulation RKIP binding to Raf-1 decreases. Serum-starved Rat-1 cells were treated with 20\% fetal calf serum for the indicated times. a, Raf-1 immunoprecipitates were immunoblotted for associated RKIP. b. Cell lysates were examined for RKIP and ERK expression. ERK activation was monitored with a phosphoERK specific antibody. vectors. Serum-starved cells were stimulated with $100 \mathrm{ng} \mathrm{ml}^{-1}$ TPA for $20 \mathrm{~min}$, and the kinase activities of Raf- 1 and HA-MEK immunoprecipitates were measured. $\mathbf{d}$, RKIP inhibits stimulation of ERK by v-Ras and v-Src. COS-1 cells were transfected with the indicated expression plasmids plus increasing amounts of RKIP. HA-ERK-2 was immunoprecipitated and assayed with MBP. e, RKIP inhibits ERK activation by BXB, but not by MEK-DD. COS- 1 cells were transfected with the indicated expression vectors and the kinase activity of HA-ERK immunoprecipitates was examined.

that suppresses background noise but strongly amplifies signals exceeding a certain threshold ${ }^{14}$. Overexpression of RKIP raises this threshold, whereas downregulation of RKIP lowers it. As the amplitude, kinetics and overall duration of ERK activity are known to vary between biological responses such as cell-cycle arrest, transformation, mitogenesis and differentiation ${ }^{1,15-18}$, RKIP is expected to exert a profound influence on these parameters.

\section{Methods}

\section{Plasmids and protein expression}

The rat RKIP $\mathrm{CDNA}^{19}$ was cloned (1) into pcDNA3 to make p353/RKIP; (2) into pCMV5 with a triple HA-tag at the amino-terminus; and (3) into pGEX-KG to make GST-RKIP. pAS-C143 encompasses RKIP nucleotides 1-429 cloned into pCMVori in antisense orientation. pCMVori contains the CMV promoter, polylinker and polyadenylation sequences from pCMV5 inserted into pUCori upstream of the polyoma virus core origin ${ }^{20}$. 6xHis-tagged MEK- and GST-fusion proteins were expressed and purified as described ${ }^{21}$. RKIP of $>95 \%$ purity was prepared from GST-RKIP by thrombin cleavage ${ }^{22}$ and subsequent FPLC separation over Superose 12.

\section{In vitro binding assays}

These contained 1-5 $\mu \mathrm{g}$ of GST-fusion protein immobilized on glutathione sepharose beads and $0.5-5 \mu \mathrm{g}$ purified recombinant protein in PBS supplemented with $10 \%$ bovine serum as a nonspecific competitor. Sf- 9 cell lysates were used as a source of Raf protein ${ }^{21}$. After incubation for $1 \mathrm{~h}$ at $4{ }^{\circ} \mathrm{C}$ the samples were washed 4 times with PBS, resolved by SDS-PAGE and blotted. The blots were developed using ECL (Amersham). 


\section{Immunoprecipitation}

For co-precipitation experiments of endogenous proteins, $2 \times 10^{7}$ Rat- 1 cells were lysed by sonication in PBS, and the immunoprecipitates were washed 4 times with PBS. Otherwise cells were lysed as described ${ }^{21}$. The antibodies used were crafVI, a peptide antibody against the 12 carboxy-terminal amino acids of Raf-1 (ref. 21); a Raf monoclonal antibody to the regulatory domain (Transduction Laboratories); anti-MEK H8 (Santa Cruz); anti-RKIP raised in rabbits immunized with purified GST-RKIP; anti-HA, 12CA5 monoclonal antibody; monoclonal anti-phospho-ERK (Sigma) and polyclonal antiphospho-MEK antibodies (New England Biolabs); and anti-GST (Pharmacia).

\section{Kinase assays}

These were done as described ${ }^{21}$. For in vitro reconstruction of the Raf/MEK/ERK cascade activated Raf- 1 was generated by co-expressing GST-Raf- 1 with v-Ras and Lck in Sf- 9 cells and collected on glutathione-sepharose beads ${ }^{21}$. Subsequent thrombin cleavage released Raf- 1 , which was fully active and $>90 \%$ pure. To activate MEK and ERK in vitro, $20 \mathrm{ng}$ of activated Raf- 1 was incubated with 40 ng of purified His/MEK-1 and $250 \mathrm{ng}$ of GST-ERK 2 in Raf kinase buffer containing $20 \mu \mathrm{M}$ ATP for $20 \mathrm{~min}$ at $30^{\circ} \mathrm{C}$. To measure kinase activities at individual steps, the respective downstream components were omitted. The activation reactions were diluted into $50 \mu \mathrm{l}$ Raf kinase buffer ${ }^{21}$ containing $20 \mu \mathrm{M}$ ATP to yield equimolar concentrations of the kinases to be assayed and incubated with increasing amounts of purified RKIP on ice for $10 \mathrm{~min}$. Then, $2 \mu \mathrm{Ci}\left[{ }^{32} \mathrm{P}\right]-\gamma$-ATP and recombinant substrates were added and incubated for $20 \mathrm{~min}$ at $30^{\circ} \mathrm{C}$. As substrates, we used $200 \mathrm{ng}$ kinase negative His/MEK-1 for Raf, $1 \mu \mathrm{g}$ kinase negative GST-ERK for MEK and $1 \mu \mathrm{g}$ GST-ELK (New England Biolabs) for ERK. In some assays $1 \mu \mathrm{g}$ GST-MEK was used as the Raf-1 substrate with identical results.

\section{Transfections}

COS- 1 cells were transfected as described ${ }^{23}$ with $2 \mu \mathrm{g}$ of HA-ERK-2, BXB, MEK and MEK DD plasmids and the indicated amounts of p353/RKIP. The total amount of transfected DNA was kept constant using the appropriate vectors as carrier DNA. NIH 3T3 and 208F cells were transfected in 6-well plates with $1 \mu \mathrm{g}$ of pCMV5-BXB and $3 \mu \mathrm{g}$ of p353/RKIP using Superfect (Qiagen). For RKIP downregulation experiments, NIH 3T3 cells were transiently cotransfected using lipofectamine with $0.5 \mu \mathrm{g}$ of $\mathrm{pHACT}^{20}$ and 1.5 or $3 \mu \mathrm{g}$ RKIP antisense expression vector (pAS-C143) or control vector (pCMVori) as indicated. pHACT expresses a truncated polyoma large-T construct with origin-binding activity, but does not bind $\mathrm{Rb}$ or $\mathrm{p} 53$, and boosts the expression of pAS-C143 to high levels. In addition, $0.1 \mu \mathrm{g}$ of an AP1-Luc reporter was transfected for reporter-gene assays. 48 hours after transfection, cells were serum starved for $20 \mathrm{~h}$ and either left untreated or treated with TPA ( $200 \mathrm{ng} \mathrm{ml}^{-1}$ ) or serum for $5 \mathrm{~h}$ before being collected. Cells were lysed and cell extracts were used for immunoblotting or assayed for luciferase activity. For the green fluorescent protein (GFP) sorting experiments, $5 \times 10^{6} \mathrm{NIH} 3 \mathrm{~T} 3$ cells were electroporated with either $100 \mu \mathrm{g}$ pCMVori, $50 \mu \mathrm{g}$ pCMV-GFP and $50 \mu \mathrm{g}$ pHACT, or $100 \mu \mathrm{g}$ pASC $143,50 \mu \mathrm{g}$ CMV-GFP and $50 \mu \mathrm{g}$ CMV-HAC. Two days later cells were trypsinized and sorted for green fluorescent cells by preparative FACS. 100,000 GFP-positive cells were lysed in SDSgel sample buffer and immunoblotted.

\section{Microinjection}

Microinjection of antibodies and reporter genes was done as described ${ }^{24,25}$. The RKIP antiserum was purified over a GST-RKIP affinity column. Cells were stained with an activation-specific anti-phospho-ERK monoclonal antibody (New England Biolabs). ERK phosphorylation was quantified by densitometry. For this purpose areas with microinjected cells were randomly photographed, and the staining intensity of whole individual cells was measured using the PcBAS software.

Received 8 April; accepted 8 July 1999.

1. Ferrell, J. E. Jr MAP kinases in mitogenesis and development. Curr. Top. Dev. Biol. 33, 1-60 (1996) 2. Morrison, D. K. \& Cutler, R. E. The complexity of Raf-1 regulation. Curr. Opin. Cell Biol. 9, 174-179 (1997).

3. Moodie, S. A., Willumsen, B. M., Weber, M. J. \& Wolfman, A. Complexes of Ras/GTP with Raf-1 and mitogen-activated protein kinase kinase. Science 260, 1658-1661 (1993).

4. Schaeffer, H. J. et al. MP1: A MEK binding partner that enhances enzymatic activation of the MAP kinase cascade. Science 281, 1668-1671 (1998).

5. Bruder, J. T., Heidecker, G. \& Rapp, U. R. Serum-, TPA-, and Ras-induced expression from Ap-1/Etsdriven promoters requires Raf-1 kinase. Genes Dev. 6, 545-556 (1992).

6. Li, S. et al. Regulation of Raf-1 kinase activity by the I4-3-3 family of proteins. EMBO J. 14, 685-696 (1995).

7. Schoentgen, F. \& Jolles, P. From structure to function: possible biological roles of a new widespread protein family binding hydrophobic ligands and displaying a nucleotide binding site. FEBS Lett. 369, 22-26 (1995).

8. Kortenjann, M. Thomae, O. \& Shaw, P. E. Inhibition of v-raf-dependent c-fos expression and transformation by a kinase-defective mutant of the mitogen-activated protein kinase Erk2. Mol. Cell. Biol. 14, 4815-4824 (1994).

9. Rapp, U. R., Troppmair, J., Beck, T. \& Birrer, M. J. Transformation by Raf and other oncogenes renders cells differentially sensitive to growth inhibition by a dominant negative c-jun mutant. Oncogene $\mathbf{9}$, 3493-3498 (1994).

10. Kolch, W. et al. Raf revertant cells resist transformation by non-nuclear oncogenes and are deficient in the induction of early response genes by TPA and serum. Oncogene 8, 361-370 (1993).

11. Catling, A. D., Schaeffer, H. J., Reuter, C. W., Reddy, G. R. \& Weber, M. J. A proline-rich sequence unique to MEK1 and MEK2 is required for raf binding and regulates MEK function. Mol. Cell. Biol. 15, 5214-5225 (1995).

12. Kolch, W. et al. Inhibition of Raf-1 signaling by a monoclonal antibody, which interferes with Raf-1 activation and with Mek substrate binding. Oncogene 13, 1305-1314 (1996).
13. Hallberg, B., Rayter, S. I. \& Downward, J. Interaction of Ras and Raf in intact mammalian cells upon extracellular stimulation. J. Biol. Chem. 269, 3913-3916 (1994).

14. Ferrell, J. E. Jr Tripping the switch fantastic: how a protein kinase cascade can convert graded inputs into switch-like outputs. Trends Biochem. Sci. 21, 460-466 (1996).

15. Tombes, R. M. et al. The mitogen-activated protein (MAP) kinase cascade can either stimulate or inhibit DNA synthesis in primary cultures of rat hepatocytes depending upon whether its activation is acute/phasic or chronic. Biochem. J. 330, 1451-1460 (1998).

16. Marshall, C. J. Specificity of receptor tyrosine kinase signaling: transient versus sustained extracellular signal-regulated kinase activation. Cell 80, 179-185 (1995).

17. Sewing, A., Wiseman, B., Lloyd, A. C. \& Land, H. High-intensity Raf signal causes cell cycle arrest mediated by p21Cip1. Mol. Cell. Biol. 17, 5588-5597 (1997).

18. Woods, D. et al. Raf-induced proliferation or cell cycle arrest is determined by the level of Raf activity with arrest mediated by p21Cip1. Mol. Cell. Biol. 17, 5598-5611 (1997).

19. Grandy, D. K. et al. Purificaton, cloning, and tissue distribution of a $23-\mathrm{kDa}$ rat protein isolated by morphine affinity chromatography. Mol. Endocrinol. 4, 1370-1376 (1990).

20. Gjorup, O. V., Rose, P. E., Holman, P. S., Bockus, B. J. \& Schaffhausen, B. S. Protein domains connect cell cycle stimulation directly to initiation of DNA replication. Proc. Natl Acad. Sci. USA 91, 1212512129 (1994).

21. Häfner, S. et al. Mechanism of inhibition of Raf-1 by protein kinase A. Mol. Cell. Biol. 14, 6696-6703 (1994).

22. Guan, K. L. \& Dixon, J. E. Eucaryotic proteins expressed in Escherichia coli: An improved thrombin cleavage and purification procedure of fusion proteins with glutathione S-transferase. Anal. Biochem. 192, 262-267 (1991).

23. Mischak, H. et al. Negative regulation of Raf-1 by phosphorylation of serine 621. Mol. Cell. Biol. 16, 5409-5418 (1996).

24. Lavbinsky, R. M. et al. Diverse signaling pathways modulate nuclear receptor recruitment of N-CoR and SMRT complexes. Proc. Natl Acad. Sci. USA 95, 2920-2925 (1998).

25. Rose, D. W., McCabe, G., Feramisco, J. R. \& Adler, M. Expression of c-fos and AP-1 activity in senescent human fibroblasts is not sufficient for DNA synthesis. J. Cell Biol. 119, 1405-1411 (1992).

26. Minden, A. et al. Differential activation of ERK and JNK mitogen-activated protein kinases by Raf-1 and MEKK. Science 266, 1719-1723 (1994).

\section{Acknowledgements}

We thank M. Karin, M. Weber, F. Hofer, P. Shaw, M. Baccarini and B. Schaffhausen for various expression plasmids; D. K. Grandy for the full-length rat cDNA homologue of RKIP; and L. Black for help with microinjections. We appreciate critical reading and helpful comments by members of the Beatson laboratories. This work was in part supported by grants of the AICR, DFG and the Sanders Stiftung to W.K. and H.M. and by an NIH grant to J.M.S.

Correspondence and requests for materials should be addressed to W.K. (e-mail: wkolch@beatson.gla. ac.uk) or J.M.S. (e-mail: john_sedivy@brown.edu).

\section{Saccharomyces cerevisiae telomerase is an Sm small nuclear ribonucleoprotein particle}

\author{
Anita G. Seto ${ }^{\star}$, Arthur J. Zaug ${ }^{\star}$, Suzanne G. Sobel $\dagger$, Sandra L. Wolin $\dagger$ \\ \& Thomas R. Cech*
}

\section{* Department of Chemistry and Biochemistry and Howard Hughes Medical Institute, University of Colorado, Boulder, Colorado 80309-0215, USA $\dagger$ Department of Cell Biology and Howard Hughes Medical Institute, Yale University School of Medicine, New Haven, Connecticut 06510, USA}

Activation of the chromosome end-replicating enzyme telomerase can greatly extend the lifespan of normal human cells ${ }^{1}$ and is associated with most human cancers ${ }^{2}$. In all eukaryotes examined, telomerase has an RNA subunit ${ }^{3}$, a conserved reverse transcriptase subunit $^{4}$ and additional proteins ${ }^{5,6}$, but little is known about the assembly of these components. Here we show that the Saccharomyces cerevisiae telomerase $\mathrm{RNA}^{7}$ has a 5' -2,2,7-trimethylguanosine (TMG) cap and a binding site for the Sm proteins, both hallmarks of small nuclear ribonucleoprotein particles (snRNPs) that are involved in nuclear messenger RNA splicing, ${ }^{8,9}$. Immunoprecipitation of telomerase from yeast extracts shows that $\mathrm{Sm}$ proteins are assembled on the RNA and that most or all of the telomerase activity is associated with the Sm-containing complex. These data support a model in which telomerase RNA is transcribed by RNA polymerase II (ref. 10) and 7-methylguanosine-capped, 